\title{
Intracellular Accumulation of Mannopine, an Opine Produced by Crown Gall Tumors, Transiently Inhibits Growth of Agrobacterium tumefaciens
}

\author{
Kun-Soo Kim, ${ }^{1}$ Chang-Ho Baek, ${ }^{1}$ Jeong Kug Lee, ${ }^{1}$ Jai Myung Yang, ${ }^{1}$ and Stephen K. Farrand ${ }^{2}$ \\ ${ }^{1}$ Department of Life Science, Sogang University, Sinsoo-Dong 1, Mapo-Gu, Seoul 121-742, Korea; \\ ${ }^{2}$ Departments of Microbiology and Crop Sciences, University of Illinois, Urbana 61801, U.S.A. \\ Submitted 17 July 2000; Accepted 27 February 2001.
}

pYDH208, a cosmid clone from the octopine-mannityl opine-type tumor-inducing (Ti) plasmid pTi15955 confers utilization of mannopine (MOP) and agropine (AGR) on Agrobacterium tumefaciens strain NT1. NT1 harboring pYDH208 with an insertion mutation in mocC, which codes for MOP oxidoreductase, not only fails to utilize MOP as a sole carbon source, but also was inhibited in its growth by MOP and AGR. In contrast, the growth of mutants with insertions in other tested moc genes was not inhibited by either opine. Growth of strains NT1 or UIA5, a derivative of $\mathrm{C58}$ that lacks pAtC58, was not inhibited by MOP, but growth of NT1 or UIA5 harboring pRE10, which codes for the MOP transport system, was inhibited by the opine. When a clone expressing $\operatorname{mocC}$ was introduced, the growth of strain NT1(pRE10) was not inhibited by MOP, although UIA5(pRE10) was still weakly inhibited. In strain NT1(pRE10, mocC), santhopine (SOP), produced by the oxidation of MOP by MocC, was further degraded by functions encoded by pAtC58. These results suggest that MOP and, to a lesser extent, SOP are inhibitory when accumulated intracellularly. The growth of NT1(pRE10), as measured by turbidity and viable cell counts, ceased upon the addition of MOP but restarted in a few hours. Regrowth was partly the result of the outgrowth of spontaneous MOP-resistant mutants and partly the adaptation of cells to MOP in the medium. Chrysopine, isochrysopine, and analogs of MOP in which the glutamine residue is substituted with other amino acids were barely taken up by NT1(pRE10) and were not inhibitory to growth of the strain. Sugar analogs of MOP were inhibitory, and those containing sugars in the $D$ form were more inhibitory than those containing sugars in the $L$ form. MOP analogs containing hexose sugars were more inhibitory than those containing sugars with three, four, or five carbon atoms. Mutants of NT1(pRE10) that are resistant to MOP arose in the zone of growth inhibition. Genetic and physiological analyses indicate that the mutations are located on pRE10 and abolish uptake of the opine.

Corresponding author: K.-S. Kim; Telephone: +82-2-705-8460; Fax: +82-2-704-3601; E-mail: kskim@ ccs.sogang.ac.kr
Crown gall tumors and hairy roots are plant neoplasias induced by pathogenic members of the genus Agrobacterium. The transformed plant cells are characterized, in part, by the production of novel, low-molecular-weight carbon compounds called "opines" (Tempé et al. 1979). The biosynthesis of these compounds in transformed plant cells is mediated by enzymes encoded by the T-DNA, which is transferred from the pathogen to the plant cell during infection (Dessaux et al. 1998; Zhu et al. 2000). In the bacterium, T-DNA forms part of the tumorinducing $(\mathrm{Ti})$ or root-inducing $(\mathrm{Ri})$ plasmids.

The opines produced by plant tumors serve as nutrient sources for the pathogenic agrobacteria. In general, the genes for the utilization of these compounds also are encoded by $\mathrm{Ti}$ or $\mathrm{Ri}$ plasmids but are not located in the $\mathrm{T}$ regions. There is a strong correlation between the types of opine produced by plant tumors and those utilized by inducing bacteria. The Opine Concept, first suggested by Petit et al. (1978; 1983), proposes that opines serve to promote selective growth of the pathogen with the specificity dictated by the catabolic genes encoded by the virulence plasmids. This model is supported by several recent studies (Guyon et al. 1993; Oger et al. 1997; Savka and Farrand 1997; Wilson et al. 1995).

Opines play additional roles in the biology of Agrobacterium spp. First, certain classes of opines induce Ti plasmid conjugal transfer (Ellis et al. 1982; Petit et al. 1978). Second, some opines enhance the acetosyringone-dependent induction of Ti plasmid-encoded virulence genes (Veluthambi et al. 1989). Third, all opines tested are attractants for Agrobacterium spp. strains (Kim and Farrand 1998). Fourth, genes coding for catabolism of the agrocinopine-type opines confer susceptibility to an antiagrobacterial antibiotic called "agrocin 84" (Ellis and Murphy 1981; Hayman and Farrand 1988; Kim and Farrand 1998). This antibiotic is produced by the nontumorigenic isolate Agrobacterium radiobacter $\mathrm{K} 84$.

The four members of the mannityl opine family can serve as sole carbon, nitrogen, and energy sources for agrobacteria harboring mannityl opine-type $\mathrm{Ti}$ or Ri plasmids. Cosmid clone pYDH208, derived from pTi15955, encodes genes for the utilization of mannopine (MOP) and its lactone, agropine (AGR). Molecular, genetic, and biochemical studies of the moc region of pYDH208 suggest that four genes, $\operatorname{agcA}, \operatorname{moc} C$, mocD, and $\operatorname{moc} E$, are necessary for the degradation of AGR and MOP in a linear pathway (Fig. 1) (Kim 
1995; Kim and Farrand 1996; Kim et al. 1996). Biochemical studies showed that MOP taken up by a specific transport system encoded by the mot genes of pTi15955 (Oger et al. 1998) or formed from AGR by the lactonase activity of MOP cyclase, the gene product of agcA (Hong et al. 1993), is oxidized to deoxyfructosyl glutamine ( $\mathrm{dfg}$ ) by MOP oxidoreductase encoded by mocC (Kim et al. 1996). The compound dfg, commonly called "santhopine" (SOP), is further catabolized to unknown intermediates by functions encoded by $m o c D$ and $m o c E$ on the Ti plasmid. This last portion of the pathway, however, is redundant because many strains of Agrobacterium spp., including C58, express a second pathway for catabolism of SOP coded for by a second very large plasmid present in these strains (Kim and Farrand 1996; Vaudequin-Dransart et al. 1998).

We observed that NT1 harboring derivatives of pYDH208 with insertions in the MOP transport system $(\operatorname{agcA}$, $\operatorname{mocD}$, or mocE) grow poorly on medium containing MOP as the sole carbon source. In contrast, NT1 harboring mocC mutants of pYDH208 failed to grow with MOP as the sole source of carbon. Furthermore, unlike any of the other mutants, the mocC mutants failed to give rise to spontaneous $\mathrm{MOP}^{+}$variants on medium containing MOP as the sole carbon source (Kim and Farrand 1996). These observations led us to consider the possibility that MOP can inhibit the growth of certain Agrobacterium spp. strains. We report here that AGR, MOP, and several of its analogs are inhibitory to the growth of Agrobacterium spp. strains that can take up these two opines but are unable to further degrade them.

\section{RESULTS}

MOP uptake is necessary for MOP sensitivity.

We tested NT1 harboring pSaB4::Tn5seq1 no. 135, which contains an insertion located in $\operatorname{mocC}$ (Fig. 2), for sensitivity to MOP, as described below. As indicated by a large zone of growth inhibition around the point at which the compound was spotted, growth of the mutant is inhibited strongly by the opine. This preliminary experiment led us to test other strains harboring plasmids with various mutations or subclones of the moc region (Table 1). Growth of strains NT1, a pTiC58-pAtC58 $8^{+}$derivative of C58, and UIA5, a plasmid-free derivative of C58, is not inhibited by MOP, whereas either strain harboring pYDH208::Tn3HoHo1 no. 11, with an insertion in $\operatorname{mocC}$ (Fig. 2), is sensitive to the opine. UIA5 harboring another mocC mutant, pSaB4::Tn5seq1 no. 135, however, is not sensitive to MOP. This strain also fails to grow, even poorly, at the expense of MOP. pSaB4::Tn5seq1 no. 135 differs from the pYDH208 derivatives in that it lacks the MOP transport genes (Figs. 1 and 2). Consistent with this observation, strains NT1 and UIA5 harboring pYDH208 with insertion no. 110, which maps to the MOP transport system, also are not sensitive to MOP. Similarly, NT1 or UIA5 harboring pYDH208 with mutations in any of the moc genes, except mocC, are not inhibited by the opine (data not shown). These results suggest that sensitivity to MOP is dependent upon opine uptake and the absence of $\operatorname{mocC}$, which codes for MOP oxidoreductase. To test this hypothesis, we examined the sensitivity of NT1 and UIA5 derivatives harboring pRE10 (Fig. 2), which en-

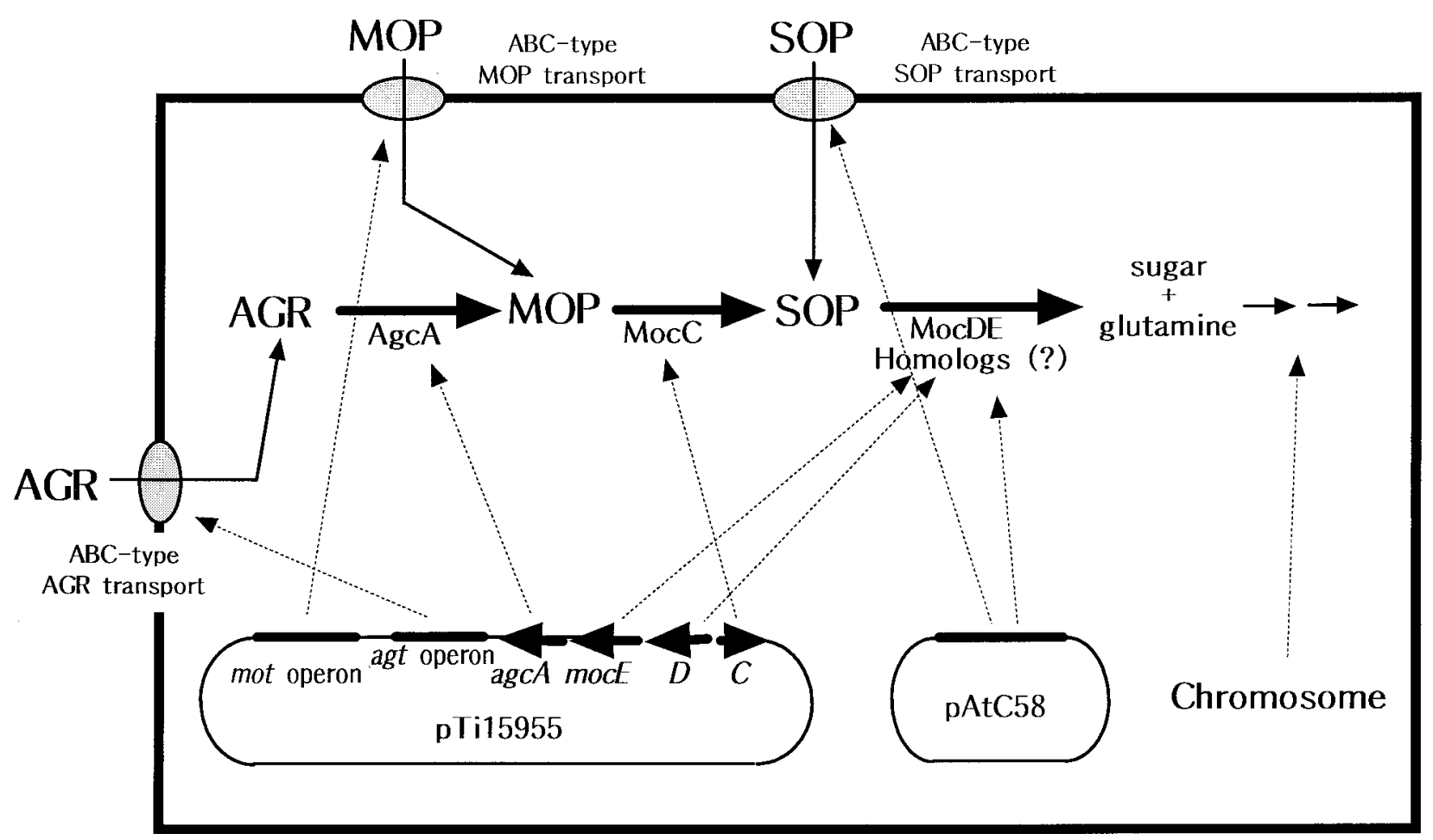

Fig. 1. Model for the degradation of mannopine (MOP) and agropine (AGR) in Agrobacterium spp. strain NT1(pTi15955). AGR, taken up by the AGR transport system encoded by the agt operon, is converted to MOP by MOP cyclase encoded by agcA. MOP taken up by its transport system or converted from AGR is oxidized to santhopine (SOP) by MocC coded for by the tumor-inducing (Ti) plasmid. SOP is further degraded by MocDE, also coded for by the Ti plasmid, or by functions encoded by megaplasmid pAtC58 in the strain. 
codes the dedicated MOP transport system (Hong and Farrand 1994; Oger et al. 1998; Zhu et al. 2000). Growth of these two strains is inhibited strongly by MOP (Fig. 3 and Table 1). Derivatives of NT1 and UIA5 harboring pSa-C, a clone of mocC, but lacking an efficient MOP transport system are not susceptible to the opine (Table 1). When we introduced pRE10 into NT1(pSa-C), growth of the resulting strain was not inhibited by MOP. Moreover, this strain efficiently utilizes MOP as a sole carbon source (Kim et al. 1996). The near-isogenic construct UIA5(pRE10, pSa-C), which lacks pAtC58, was inhibited by MOP but to a lesser extent than was UIA5(pRE10). In summary, those strains which can take up MOP but lack mocC are most sensitive to MOP. These results suggest that MOP is inhibitory to growth and that inhibition requires intracellular accumulation of the opine.

We also tested these strains for growth inhibition by AGR. This lactone opine is catabolized by a pathway that proceeds through MOP (Fig. 1), but AGR is taken up by its own transport system (Fig. 2) (Hong 1994; Hong et al. 1993; Zhu et al. 2000). We assessed the sensitivity levels of several strains to MOP and AGR by determining the minimal concentration of these opines, which resulted in a visibly detectable zone of growth inhibition. The tested strains were more susceptible to MOP than to AGR (Fig. 3), and the minimal inhibitory concentration (MIC) value for AGR was 100 nmole, whereas the MIC for MOP was as low as 0.1 nmole (Table 2).

\section{Inhibitory activity of MOP analogs.}

To examine the chemical attributes of MOP that are associated with growth-inhibition activity, we tested analogs of the opine against strain UIA5(pRE10) (Fig. 4 and Table 3). In addition, we performed tests on NA, a rich medium, and on ATNG minimal medium to determine whether medium composition has an effect on the inhibition exerted by MOP and its analogs. The inhibitory effects of MOP and its analogs were slightly weaker when assessed on NA medium compared with ATNG minimal medium (data not shown). All of the analogs in which mannose was replaced with another sugar were inhibitory to growth of UIA5(pRE10) (Table 3). SOP, the immediate catabolic breakdown product of MOP, however, is significantly less inhibitory compared with the mannityl opine. Mannopinic acid (MOA), a natural amino acid analog of MOP, also is inhibitory but less so compared with MOP. Amino acid analogs of MOP in which the glutamine is replaced with valine, asparagine, or methionine did not detectably inhibit growth of the strain.

Strains NT1 and NT1(pRE10) were tested for their capacity to take up MOP, its sugar, and amino acid analogs as well as SOP; its lactone, chrysopine; and its lactam, isochrysopine. Strain NT1 could take up SOP, but failed to take up significant amounts of the other compounds tested, including chrysopine, the lactonized derivative of SOP (Fig. 5). Strain NT1(pRE10), however, removed most of the compounds from the medium, although this strain also failed to take up significant amounts of chrysopine or isochrysopine and, compared with MOP, took up reduced amounts of glucopine, D-Ara-Gln, L-Xyl-Gln, DMan-Val, D-Man-Asn, and D-Man-Met. Neither strain took up detectable amounts of MOA (data not shown).

\section{Octopine is inhibitory to growth of strains containing the opine transport system but lacking catabolic genes.}

To test whether growth inhibition is specific to the mannityl opines, we tested octopine for its activity on derivatives of the octopine-mannityl opine-type strain R10. Growth of strains KYC8 and KYC16, which have mutations in the two genes coding for octopine oxidase but express the octopine transport system, was inhibited by octopine at an MIC of 100 nmoles. In contrast, the growth of KYC11, a mutant with an insertion in a gene of the octopine transport system, was not inhibited by the opine (data not shown). These results suggest that octopine also is inhibitory to growth when accumulated intracellularly.

\section{Growth inhibition of NT1(pRE10) by MOP is transient.}

The addition of MOP to an exponentially growing culture of NT1(pRE10) in ATNG liquid minimal medium led to an immediate cessation of growth (Fig. 6A). Culture growth resumed in a few hours, however, although the cells grew at a slightly slower rate and to a final cell density at a stationary phase that was approximately threefold lower than those cells grown in medium lacking MOP (Fig. 6B). Whereas the addition of MOP inhibited growth, it did not result in a significant

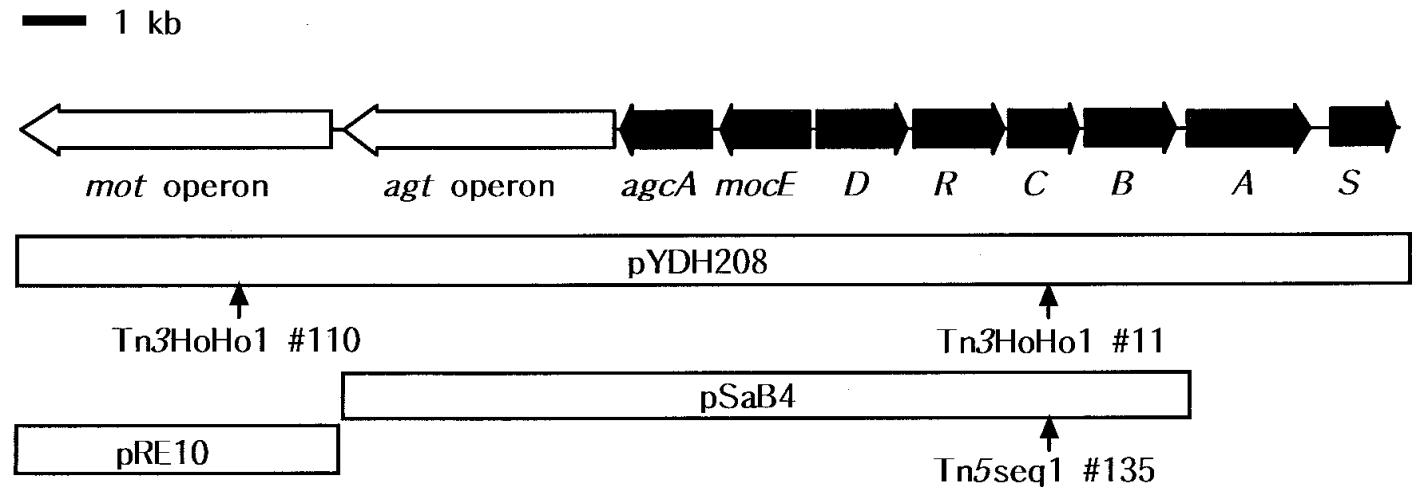

Fig. 2. Organization of genes associated with the utilization of mannopine (MOP) and agropine (AGR) on pYDH208 and its subclones from pTi15955. Plasmid pYDH208 contains a 21-kb fragment, which contains all of the genes responsible for the transport and degradation of MOP and AGR (Hong et al. 1993). Plasmid pSaB4 contains a 14-kb BamHI fragment of pYDH208, which contains the agt genes for the transport of AGR, and genes essential for the degradation of MOP and AGR (Kim and Farrand 1996; Kim et al. 1995). Plasmid pRE10 contains all of the genes required for the transport of MOP (mot genes) (Oger et al. 1998). The positions of the transposon insertions in derivatives of pYDH208 and pSaB4 employed in this study are indicated by vertical arrows. 
drop in the number of culturable cells in the population (Fig. $6 \mathrm{C})$. Thus, the opine, which inhibited growth of the cells, is not bactericidal.

\section{Analysis of spontaneous MOP-resistant mutants isolated from UIA5(pRE10).}

Spontaneous mutants resistant to inhibition by MOP, galactopine, and SOP appeared in the zone of growth inhibition 1 week after incubation on NA plates. On ATNG medium, the appearance of this type of mutant was delayed up to 2 weeks. Six spontaneous MOP-resistant mutants of NT1(pRE10) were isolated at random. The plasmid from each, named pRE10r-1 through -6 , was isolated, reintroduced into wild-type NT1, and the resulting strains were tested for susceptibility to MOP. None of these constructs were inhibited by the opine (data not shown), nor could these strains take up the opine from the medium (Fig. 7). Furthermore, when these plasmids were introduced into strain UIA5( $\mathrm{pSaB} 4)$, the resulting cells failed to grow on medium containing MOP as the sole carbon source (data not shown). In a second set of experiments, the pRE10r plasmids from the original MOP-resistant derivatives were cured, and the resulting strains, named NT1c-1 through -6 , were retransformed with wild-type pRE10. The resulting constructs, NT1c-1(pRE10) through -6(pRE10), were sensitive to MOP and could take up the opine from the medium (Fig. 7 and data not shown). These results indicate that mutations resulting in resistance to MOP are located on pRE10. In an attempt to locate the mutations, we subjected each of the pRE10r plasmids to digestion with several restriction endonucleases. The fragment profiles of the six mutant plasmids were indistinguishable from those of the wild-type parent plasmid (data not shown). The pRE10r plasmids also were introduced into UIA5 harboring a clone coding for the wild-type MOP transport genes. Growth of the transformant cells was still inhibited by MOP (data not shown), indicating that the mutations in the pRE10r plasmids are recessive.

\section{Incidence of MOP-resistant mutants in populations of UIA5(pRE10).}

We determined which proportion of the cells in a population are resistant to MOP as well as the influence of growth in the presence of the opine on this subpopulation. Strain UIA5(pRE10) was grown to late exponential phase in ATNG medium, with and without MOP, at a final concentration of 1 $\mathrm{mM}$. A sample of each culture was removed, suitably diluted, and volumes were plated onto ATNG media, for viable counts, and ATNG media containing MOP to assess the number of opine-resistant cells in the population. In addition, a volume of each of these cultures was used to inoculate a second set of cultures in ATNG medium and ATNG medium containing MOP. These two sets of secondary cultures were grown, sampled, and the cells were plated onto ATNG medium and ATNG medium containing MOP, as described above. The primary culture grown in the absence of MOP yielded approximately one MOP-resistant colony out of $10^{5}$ viable cells, whereas in the culture grown in the presence of the opine, MOP-resistant cells were approximately 400 times more abundant (Table 4). Consistent with these low frequencies, of the 20 colonies tested that arose on ATNG-viable titer plates from the two cultures, none were resistant to MOP, as assessed by the plateoverlay method (data not shown). The secondary culture that was started from cells taken from unsupplemented ATNG yielded approximately tenfold higher numbers of MOPresistant colonies compared with the primary culture (Table

Table 1. Growth inhibitory effect of mannopine (MOP) on various derivatives of Agrobacterium tumefaciens NT1

\begin{tabular}{|c|c|c|c|c|c|c|}
\hline \multirow[b]{2}{*}{ Strains } & \multicolumn{3}{|c|}{ Relevant genotypes } & \multirow[b]{2}{*}{ Growth on MOP $^{b}$} & \multirow[b]{2}{*}{ Sensitivity to MOP $^{c}$} & \multirow[b]{2}{*}{ Resistant mutants } \\
\hline & pAtC58 & $\operatorname{moc} C$ & $\operatorname{mot}^{\mathrm{a}}$ & & & \\
\hline NT1 & + & - & - & - & - & $\mathrm{NA}^{\mathrm{e}}$ \\
\hline NT1(pSaB4) & + & + & - & \pm & - & NA \\
\hline NT1(pYDH208) & + & + & + & ++ & - & NA \\
\hline NT1(pRE10) & + & - & + & - & ++ & + \\
\hline NT1(pSaB4::Tn5seq1 no. 135$)$ & + & - & - & - & + & - \\
\hline NT1(pYDH208::Tn3HoHo1 no. 11) & + & - & + & - & ++ & - \\
\hline $\mathrm{NT} 1(\mathrm{pSa}-\mathrm{C})$ & + & + & - & ++ & - & NA \\
\hline NT1(pRE10, pSa-C) & + & + & + & ++ & - & NA \\
\hline NT1(pYDH208::Tn5 no. 110) & + & + & - & \pm & - & NA \\
\hline UIA5 & - & - & - & - & - & NA \\
\hline $\mathrm{UIA5}(\mathrm{pSaB} 4)$ & - & + & - & - & - & NA \\
\hline UIA5(pYDH208) & - & + & + & ++ & - & NA \\
\hline UIA5(pRE10) & - & - & + & - & ++ & ++ \\
\hline UIA5(pSaB4::Tn5seq1 no. 135$)$ & - & - & - & - & - & NA \\
\hline UIA5(pYDH208::Tn3HoHo1 no. 11) & - & - & + & - & ++ & ++ \\
\hline $\mathrm{UIA} 5(\mathrm{pSa}-\mathrm{C})$ & - & + & - & - & - & NA \\
\hline UIA5(pRE10, pSa-C) & - & + & + & - & + & - \\
\hline UIA5(pYDH208::Tn5 no. 110) & - & + & - & - & - & NA \\
\hline
\end{tabular}

${ }^{\mathrm{a}} \mathrm{mot}=\mathrm{MOP}$ transport operon (Oger et al. 1998).

${ }^{\mathrm{b}}$ Growth was examined after 3 days of incubation on solid minimal medium containing MOP as the sole carbon source; - , no growth; \pm , slow growth; + , wild-type growth; ++ , growth faster than wild type.

${ }^{\mathrm{c}}$ Cells suspended in prewarmed $0.6 \%$ agar solution were spread on top of a plate containing solid ATNG medium supplemented with the appropriate antibiotics. A 1- $\mu$ l volume of $100 \mathrm{mM}$ MOP was spotted at the center of the plate. Cultures were incubated for 1 week, and growth inhibition around the spot was observed visually. Inhibition of growth was scored as - , no growth inhibition; +, diameter of inhibition zone at $10-30$ mm; ++ , diameter of inhibition zone $>30 \mathrm{~mm}$; and NA, not applicable (see text for details).

${ }^{\mathrm{d}}$ Spontaneous resistant mutants appeared as small colonies growing within the zones of growth inhibition following a prolonged incubation (up to 2 weeks); -, no appearance of mutants; +, appearance of a small number of mutants; ++, appearance of large numbers of mutants.

e Not applicable. 
4). When cells grown in MOP-supplemented medium were recultured in ATNG, approximately seven in 1,000 colonies was resistant to MOP, a proportion similar to that of the inoculum (Table 4). Again, all 20 colonies recovered from the two sets of ATNG plates used for viable counts were inhibited by MOP when tested by the plate assay (data not shown). When recultured in ATNG containing MOP, however, cells grown previously in medium supplemented with the opine showed a marked increase in the proportion of colonies resistant to MOP, and about three in ten grew on medium containing the opine (Table 4). Consistent with this increase, of the 20 colonies picked from the ATNG-viable count plates inoculated with the secondary culture grown in ATNG containing MOP, three were resistant to the opine when tested with the plateoverlay assay (data not shown).

\section{DISCUSSION}

In this report, we show that AGR, MOP, and MOP analogs are inhibitory to the growth of some Agrobacterium spp. strains. Derivatives of strains UIA5 or NT1, which can take up MOP but lack mocC, are sensitive to the opine. This suggests that MOP, rather than a breakdown product of the opine, is the inhibitory factor. SOP is much less inhibitory to NT1(pRE10) than is MOP (Fig. 4 and Table 3). Strain
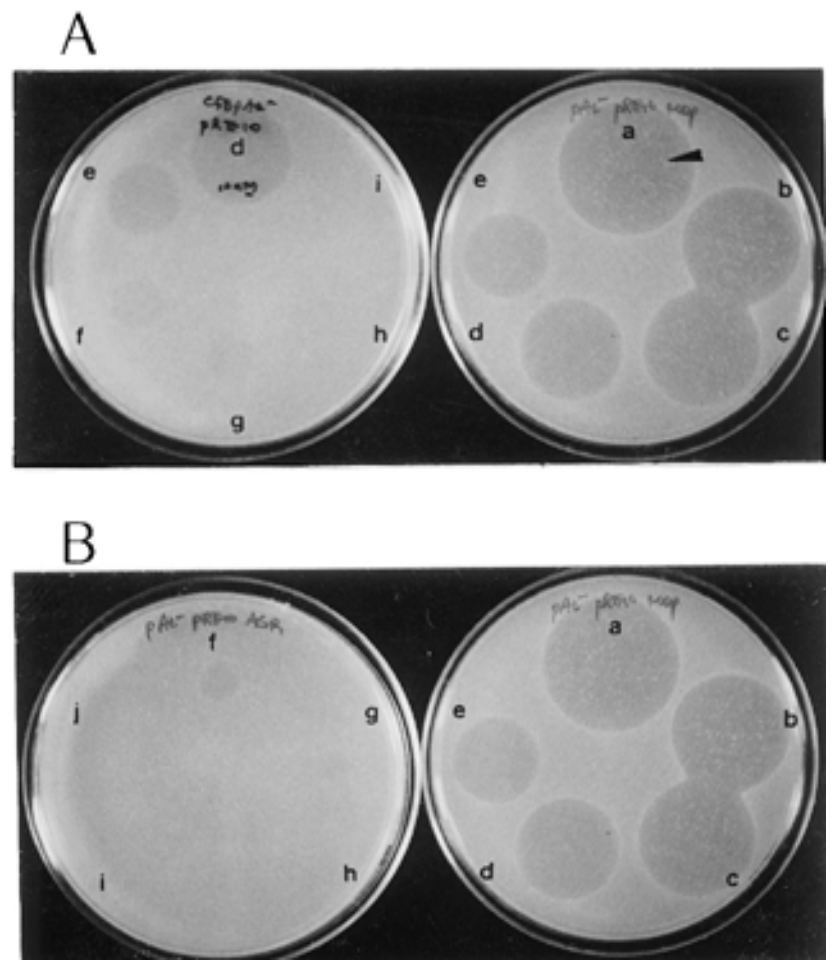

Fig. 3. Mannopine (MOP) and agropine (AGR) inhibit growth of certain agrobacterial strains. Growth inhibition of UIA5(pRE10) by MOP (right plate) and AGR (left plate). Volumes of $1 \mu \mathrm{l}$ of MOP (a-e) or AGR (f-i) were spotted onto the soft agar overlays at the following amounts, per $\mu \mathrm{l}$ : a and f, 200 nmole; $\mathrm{b}$ and $\mathrm{g}, 100$ nmole; $\mathrm{c}$ and h, 50 nmole; $\mathrm{d}$ and i, 10 nmole. B, Growth inhibition of UIA5(pYDH208::Tn3HoHo1 no. 11) by MOP (right plate) and AGR (left plate), per $\mu$ l: $a$ and f, 200 nmole; $b$ and $\mathrm{g}, 100$ nmole; e and h, 50 nmole; d and i, 10 nmole; e and j, 5 nmole. Spontaneous MOP-resistant mutants arising within the zone of growth inhibition are indicated by an arrowhead.
UIA5(pRE10) transports MOP and SOP but cannot degrade either of these opines (Hong and Farrand 1994; Kim 1995). UIA5(pRE10, pSa-C), which can degrade MOP to SOP, is still sensitive to MOP. The oxidation of MOP to SOP by MOP oxidoreductase is much slower than the reverse reaction of the enzyme (Kim et al. 1996), raising the possibility that MOP accumulates in these cells. NT1(pRE10, pSa-C) and NT1(pSa$\mathrm{C})$, however, are resistant not only to MOP, but also grow well with MOP as a sole carbon source (Kim and Farrand 1996). We suggest that, in these NT1 derivatives, SOP produced from MOP by MOP oxidoreductase is further degraded by the SOP utilization system coded for by the megaplasmid pAtC58 (Fig. 1) (Kim and Farrand 1996; Kim et al. 1996; VaudequinDransart et al. 1995). Supporting this hypothesis, we recently subcloned a region from the megaplasmid that confers utilization of MOP on UIA5 carrying a mocC clone (Baek et al. 2000). In strain NT1(pRE10, pSa-C), the additional metabolism of SOP presumably shifts the equilibrium of MOP oxidoreductase in favor of the oxidation reaction.

AGR is inhibitory to the growth of strains UIA5 and NT1 harboring pYDH208 with an insertion in $\operatorname{mocC}$ (Fig. 3). This clone codes for an intact AGR transport system and the enzyme required to convert AGR to MOP. The lactone is less inhibitory than MOP on a molar basis. We propose that growth inhibition by AGR results from the slow conversion of the lactone to MOP mediated by MOP cyclase. This suggests, in turn, a physiological role for MOP cyclase. We previously proposed that MOP cyclase rapidly converts MOP to AGR to
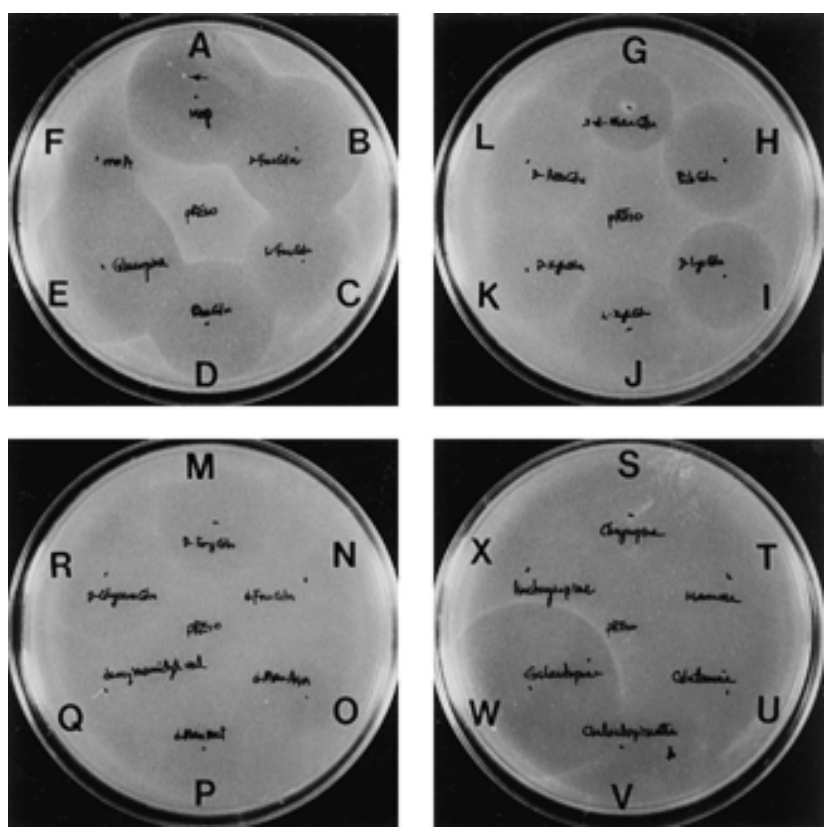

Fig. 4. Inhibitory effect of mannopine (MOP) and its analogs on the growth of UIA5(pRE10). Volumes of $1 \mu \mathrm{l}$ of MOP or its analogs (200 nmole/ $\mu \mathrm{l}$ ) were spotted onto the surface of soft agar overlays on ATNG minimal medium containing $100 \mu \mathrm{g}$ of carbenicillin per $\mathrm{ml}$, as described in text. A, MOP; B, D-Fuc-Gln; C, L-Fuc-Gln; D, L-Rha-Gln; E, glucopine; F, mannopinic acid; G, deoxy-MOP; H, D-Rib-Gln; I, D-LyxGln; J, L-Xyl-Gln; K, D-Xyl-Gln; L, D-Ara-Gln; M, D-Ery-Gln; N, DFru-Gln (santhopine); O, D-Man-Asn; P, D-Man-Met; Q, D-Man-Val; R, D-Glycero-Gln; S, chrysopine; T, mannose; U, glutamine; V and W, galactopine; $\mathrm{X}$, isochrysopine. Arrow marks the position of a spontaneous MOP-resistant mutant. 
sequester the opine as its lactone (Hong and Farrand 1994; Kim and Farrand 1996). It also is possible that this conversion serves to reduce the intracellular concentration of MOP to levels that do not inhibit cell growth. In this scenario, MOP transported into the bacteria by the MOP transport system is converted by MOP cyclase to AGR, which is much less inhibitory. AGR is then converted back to MOP by MOP cyclase, but only when it can be further metabolized by MOP oxidoreductase. The concomitant oxidation of MOP to SOP by MOP oxidoreductase insures that the intracellular concentration of MOP does not reach inhibitory levels.

Inhibitory activity depends upon the sugar and amino acid moieties. Analogs in which the sugar substitute is in the D form are slightly more inhibitory than those in which the sugar is in the L form. Furthermore, analogs with six-carbon sugar substituents are more inhibitory than those with five-, four-, or three-carbon sugar substituents. In addition, the presence of a hydroxyl group at the C-2 position of the sugar residue is important for the inhibitory effect. Finally, the glutamine residue of MOP is important for the effect, and analogs containing glutamate, asparagine, valine, or methionine either lack activity or are considerably less inhibitory compared with MOP.

The role of the amino acid residue and the hydroxyl group of sugar residue may be indirect. MOP analogs containing valine, asparagine, or methionine are poorly taken up by NT1(pRE10), and the chrysopine family of opines, all of which lack the hydroxyl group at the C-2 position of the sugar residue, are not taken up by the MOP or AGR transport systems. Even weak transport can lead to inhibition, however, and glucopine strongly inhibits growth, even though it is poorly taken up by the strain.

Among the deoxyaldityl analogs, glucopine, galactopine, and deoxy-MOP are lactonized by MOP cyclase (Hong and Farrand 1994). None of the amino acid analogs, including MOA, are recognized by the lactonizing enzyme. Among the sugar analogs of MOP, only D-Lyx-Gln served as a substrate for MOP oxidoreductase (Kim et al. 1996). With the exception of D-Man-Asn, none of the amino acid analogs of MOP we tested could be oxidized significantly by the enzyme (Kim et al. 1996). Furthermore, most of the analogs cannot support the

Table 2. Inhibitory effects of mannopine (MOP) and agropine (AGR) on the growth of Agrobacterium tumefaciens strains

\begin{tabular}{|c|c|c|c|c|c|c|c|c|c|c|c|c|c|}
\hline \multirow[b]{2}{*}{ Strain $^{\mathbf{b}}$} & \multirow[b]{2}{*}{ Opine } & \multicolumn{10}{|c|}{ Diameter $(\mathrm{cm})$ of zones of growth inhibition around opine spots $\left(\right.$ nmoles $\left.^{\mathrm{a}}\right)$} & \multirow[b]{2}{*}{ MIC $^{\mathbf{c}}$} & \multirow[b]{2}{*}{ Appearance of resistant mutant } \\
\hline & & 200 & 100 & $\mathbf{5 0}$ & 10 & 5 & 2 & 1 & 0.2 & 0.1 & $\mathbf{0}$ & & \\
\hline \multirow{2}{*}{ NT1(pRE10) } & MOP & 4 & 3.7 & 3.1 & 2.7 & 2.4 & 1.8 & 1.3 & 0.8 & 0.7 & 0 & $<0.1$ & + \\
\hline & AGR & 0.8 & 0.9 & 0 & 0 & 0 & 0 & 0 & 0 & 0 & 0 & 100 & - \\
\hline \multirow[t]{2}{*}{ UIA5(pRE10) } & MOP & 3.2 & 2.7 & 2.8 & 2.3 & 1.7 & 1.2 & 1.1 & 0.5 & 0 & 0 & 0.2 & ++ \\
\hline & AGR & 0.8 & 0.7 & 0 & 0 & 0 & 0 & 0 & 0 & 0 & 0 & 100 & - \\
\hline
\end{tabular}

a Tested on solid ATNG containing appropriate antibiotics. A 1- $\mu 1$ volume containing the amount of MOP or AGR indicated was spotted onto the overlays. Diameter of the zones of growth inhibition was measured 1 week after spotting.

${ }^{\mathrm{b}}$ NT1 and UIA5 were not susceptible to MOP or AGR at $200 \mathrm{nmole} / \mu \mathrm{l}$.

${ }^{\mathrm{c}}$ Minimal inhibitory concentration (MIC) is defined as the minimal amount of opine applied which generated a detectable zone of growth inhibition.

Table 3. Mannopine (MOP) analogs and their growth inhibitory properties

\begin{tabular}{|c|c|c|}
\hline MOP analogs & Common name or abbreviation & Growth inhibition activity $^{\mathrm{a}}$ \\
\hline $\begin{array}{l}\text { Deoxyaldityl analogs } \\
N \text {-1-(1-deoxy-D-glucityl)-L-glutamine } \\
N \text {-1-(1-deoxy-D-galacityl)-L-glutamine } \\
N \text {-1-(1-deoxy-D-galacityl)-L-glutamine } \\
N \text {-1-(1-deoxy-L-rhamnityl)-L-glutamine } \\
N \text {-1-(1-deoxy-L-fucityl)-L-glutamine } \\
N \text {-1-(1-deoxy-D-fucityl)-L-glutamine } \\
N \text {-1-(1,2-dideoxy-D-mannityl)-L-glutamine } \\
N \text {-1-(1-deoxy-D-arabinityl)-L-glutamine } \\
N \text {-1-(1-deoxy-D-xylityl)-L-glutamine } \\
N \text {-1-(1-deoxy-L-xylityl)-L-glutamine } \\
N \text {-1-(1-deoxy-D-lyxityl)-L-glutamine } \\
N-1-(1-d e o x y-D-r i b i t y l)-L-g l u t a m i n e \\
N-1-(1-\text { deoxy-D-erythrityl)-L-glutamine } \\
N \text {-1-(2,3-dihydroxypropyl)-L-glutamine }\end{array}$ & $\begin{array}{l}\text { Glucopine } \\
\text { Galactopine } \\
\text { Galactopinic acid } \\
\text { L-Rha-Gln } \\
\text { L-Fuc-Gln } \\
\text { D-Fuc-Gln } \\
\text { deoxy-MOP } \\
\text { D-Ara-Gln } \\
\text { D-Xyl-Gln } \\
\text { L-Xyl-Gln } \\
\text { D-Lyx-Gln } \\
\text { D-Rib-Gln } \\
\text { D-Ery-Gln } \\
\text { D-glycero-Gln }\end{array}$ & $\begin{array}{l}++ \\
++ \\
\pm \\
+ \\
+ \\
++ \\
\pm \\
+ \\
+ \\
+ \\
+ \\
+ \\
+ \\
+\end{array}$ \\
\hline $\begin{array}{l}\text { Amino acid analogs } \\
N \text {-1-(1-deoxy-D-mannityl)-L-glutamate } \\
N \text {-1-(1-deoxy-D-mannityl)-L-valine } \\
N \text {-1-(1-deoxy-D-mannityl)-L-aspargine } \\
N \text {-1-(1-deoxy-D-mannityl)-L-methionine }\end{array}$ & $\begin{array}{l}\text { MOA }^{\mathrm{b}} \\
\text { D-Man-Val } \\
\text { D-Man-Asn } \\
\text { D-Man-Met }\end{array}$ & $\begin{array}{l}+ \\
- \\
- \\
-\end{array}$ \\
\hline $\begin{array}{l}\text { Chrysopine family } \\
N \text {-1-(1-deoxy-D-fructosyl)-L-glutamine } \\
N \text {-1-(1-deoxy-D-fructopyranosyl)-L- glutamine- } \delta \text {-lactone } \\
N \text {-1-(1-deoxy-D-fructosyl)-5-oxo-L-proline }\end{array}$ & $\begin{array}{l}\text { Santhopine } \\
\text { Chrysopine } \\
\text { Isochrysopine }\end{array}$ & $\begin{array}{l} \pm \\
- \\
-\end{array}$ \\
\hline
\end{tabular}

${ }^{a}$ Inhibitory effect was examined after applying $1 \mathrm{mM}$ substrates with UIA5(pRE10). - , no growth inhibition; \pm , zone of inhibition measuring < $10 \mathrm{~mm}$; + , diameter of zone of inhibition of $10-30 \mathrm{~mm} ;++$, diameter of zone of inhibition at $>30 \mathrm{~mm}$.

${ }^{\mathrm{b}}$ Mannopinic acid (MOA) is a natural member of the mannityl opine family. 
growth of wild-type strain 15955. It appears that the capabilities of MOP analogs to serve as substrates for the two enzymes do not correlate directly with the inhibitory activities of the compounds.

In a previous report, we suggested that megaplasmid pAtC58, which is present in strain NT1, codes for a system that can transport MOP. Hence, NT1 harboring pSaB4, a moc region clone that lacks the MOP transport system, can grow on medium containing MOP as the sole carbon source (Kim et al. 1996). The capacity of the strain to take up MOP explains why $\mathrm{NT} 1(\mathrm{pSaB} 4:: \operatorname{Tn} 5 \mathrm{seq} 1$ no. 135) in which mocC is disrupted is inhibited by the opine. UIA5 $(\mathrm{pSaB} 4)$, which lacks pAtC58 and its SOP transport system, does not utilize MOP unless pRE10, which encodes the MOP transport system, also is present in the strain (Kim and Farrand 1996). The observation that pRE10 derivatives isolated from MOP-resistant mutants do not allow NT1 to take up MOP supports out hypothesis that the opine must be taken up by a specific transporter to inhibit growth.

Results from our growth experiments indicate that inhibition by MOP is transient and bacteriostatic. Resumption of growth apparently results from two phenomena, physiological adaptation and mutations in the MOP transport system. Clearly such transport mutants are present at low levels in the population of cells, even when grown in the absence of the inhibitory opine (Table 4). A single culture cycle in medium containing MOP, however, does not enrich for these mutants at a level that can account for the overall growth of the population. Thus, we conclude that, after an inhibitory phase, the sensitive members of the population can adapt to the opine and resume growth. Dilution and regrowth of this culture in medium containing MOP, however, does result in a substantial enrichment for resistant mutants (Table 4), suggesting that the sensitive members of the population go through a second inhibitory phase in the new culture.

How MOP inhibits growth of the bacteria remains to be determined. The effect is not confined to this opine: octopine also inhibits growth of cells that can take up but not degrade
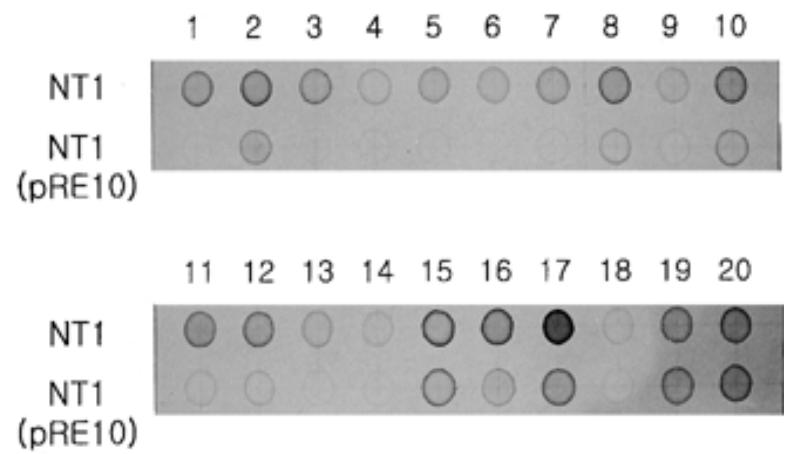

Fig. 5. Uptake of mannopine (MOP) analogs by NT1(pRE10). Cultures of NT1 and NT1(pRE10) were grown for $30 \mathrm{~h}$ in ATNG liquid media containing one of each of the MOP analogs at a final concentration of 1 $\mathrm{mM}$. Cells were removed by centrifugation, culture supernatants were spotted onto Whatman 3MM filter paper (Kent, U.K.), and the substrates remaining in the medium were visualized by alkaline silver nitrate staining, as described in text. 1, MOP; 2, glucopine; 3 , galactopine; 4, galactopinic acid; 5, L-Rha-Gln; 6, L-Fuc-Gln; 7, D-Fuc-Gln; 8, D-Ara-Gln; 9, D-Xyl-Gln; 10, L-Xyl-Gln; 11, D-Lyx-Gln; 12, D-Rib-Gln; 13, DEry-Gln; 14, D-Glycero-Gln; 15, D-Man-Val; 16, D-Man-Asn; 17, DMan-Met; 18, santhopine; 19, chrysopine; 20, isochrysopine. the arginyl opine. Moreover, agrocinopines A and B, members of a class of sugar phosphodiesterase opines, also inhibit the growth of strains that can transport but not catabolize these compounds (Hayman et al. 1993). Growth inhibition by these opines resembles the phenomenon of abortive metabolism in which simple substrates such as glucose and rhamnose inhibit the growth of mutants blocked in the catabolism of these compounds (Barkulis 1949; Böck and Neidhardt 1966; Fradkin and Fraekel 1971; Irani and Maitra 1977). Abortive
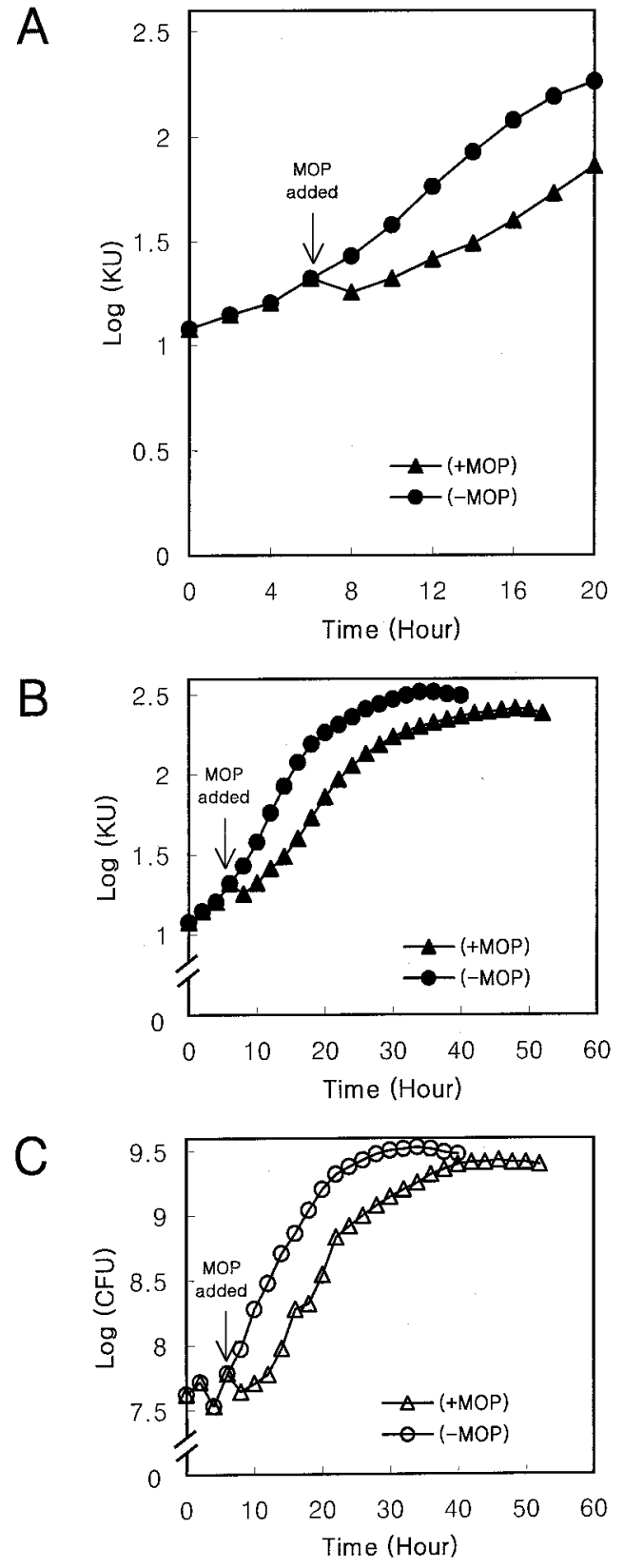

Fig. 6. Mannopine (MOP) transiently inhibits growth of NT1(pRE10). Strain NT1(pRE10) was cultured in ATNG medium to a population density corresponding to a Klett value (red filter) of approximately 20 . The culture was split into two, MOP was added to one subculture, and the two cultures were incubated in parallel. Growth was followed by Klett colorimetry (A and B), and viable population size was determined by dilution plate counts (C) at 2-h intervals. A, The first $20 \mathrm{~h}$ of growth of the culture shown in $\mathbf{B}$. 
metabolism often is bactericidal, whereas the effect of MOP on the growth of strains is bacteriostatic. Moreover, abortive metabolism is linked to the formation and accumulation of phosphorylated intermediates of pathways of central metabolism (Böck and Neidhardt 1966). Neither MOP nor SOP are substrates of central metabolism but must be further degraded, probably to fructose and glutamine, by a plasmid-encoded, opine-specific pathway (Kim et al. 1996). Furthermore, the MOP transport system is a member of the periplasmic proteindependent ABC family of transporters (Oger et al. 1998), and uptake does not involve phosphorylation of the opine. Our analyses of cell extracts by high-voltage paper electrophoresis indicate that the intracellular opine remains in its original form (Kim 1995). Finally, whereas the MOP catabolism system codes for a protein that could be a kinase (Kim et al. 1996), mocE, the gene for this protein, is not required for growth inhibition by the opine. Thus, it is not clear how accumulation of these opines or their immediate breakdown products could interfere with the central pathways of anabolic or catabolic metabolism. It is possible, however, that accumulation of these compounds alters the internal osmotic balance of the cells. Adaptation to such an imbalance or activation of an efflux system, which is able to export the opine (Decker et al. 1993), could account for the transient nature of the inhibition.

The Opine Concept describes a positive role for opines in which these compounds make the rhizosphere environment favorable for growth of agrobacteria that can utilize these substrates. It is conceivable that opines also may play a negative role by inhibiting the growth of competitor soil bacteria, which can take up the compounds but cannot degrade them. In this way, opines may contribute to stable colonization by agrobacteria of a host plant bearing tumors producing the compounds. At the moment, we do not know whether such opine-sensitive bacteria exist in nature. Screening various Agrobacterium spp. isolates or other soil bacteria for sensitivity to MOP or its analogs, however, could provide information concerning the significance of the inhibitory properties of MOP in the plant-Agrobacterium spp. relationship.

\section{MATERIALS AND METHODS}

\section{Bacterial strains and plasmids.}

Strains and plasmids used in this study are listed in Table 5. Plasmid pRE10 contains a MOP transport system cloned from pYDH208 in the broad host range vector pRK415K (Hong

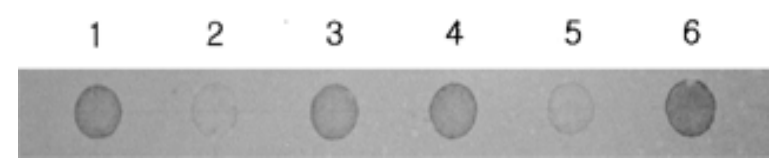

Fig. 7. Mannopine (MOP)-insensitive mutants of NT1(pRE10) fail to take up the opine. Strains were grown in $0.5 \mathrm{ml}$ of ATNG containing MOP for $30 \mathrm{~h}$, cells were removed from the culture by centrifugation, and the culture supernatants were assessed for any remaining amounts of MOP by visualization with alkaline silver nitrate, as described in the text. Spots represent supernatants from cultures of: 1, NT1;2, NT1(pRE10); 3, primary mutant NT1(pRE10r-1); 4, NT1 transformed with pRE10r-1; 5, NT1c-1 retransformed with pRE10; and 6, NT1c-1 retransformed with pRE10r-1. and Farrand 1994). This plasmid constitutively expresses a MOP transport system (Hong and Farrand 1994). Plasmid pSaB4 contains a 14-kb Bam HI fragment from pYDH208 that codes for all of the functions necessary for the enzymatic degradation of AGR and, therefore, of MOP but does not code for a MOP transport system (Fig. 1). Plasmids pSaB4::Tn5seq1 no. 135 and pYDH208::Tn3HoHo1 no. 11 contain transposon insertions in the mocC gene (Kim and Farrand 1996). Strains KYC8 and KYC16 are derivatives of A. tumefaciens R10 with mutations in ooxA and ooxB, respectively (Cho et al. 1996). These two Ti plasmid genes code for subunits of octopine oxidase that is responsible for the first step of octopine degradation. Strain KYC11 has an insertion in occ $Q$ that codes for a subunit of the octopine transport system (Cho et al. 1996).

\section{Culture media.}

Nutrient broth (NB; Difco, Detroit, MI, U.S.A.) and LuriaBertani broth (LB; GIBCO-BRL, Rockville, MD, U.S.A.) were used as rich media. NB or AT minimal medium supplemented with $0.15 \%\left(\mathrm{NH}_{4}\right)_{2} \mathrm{SO}_{4}$ (ATN) (Dessaux et al. 1987) and glucose at $5 \mathrm{mM}$ (ATNG) were used to grow agrobacteria for sensitivity tests. Noble agar (Difco) was used (final concentration $1.8 \%$ ) to solidify the minimal medium. A. tumefaciens strains were grown at $28^{\circ} \mathrm{C}$.

\section{Chemicals.}

MOP was synthesized as described previously (Petit et al. 1983). Stock solutions were prepared in distilled water at 100 $\mathrm{mM}$, sterilized by filtration, and kept frozen at $-20^{\circ} \mathrm{C}$. AGR was synthesized enzymatically from MOP (Dessaux et al. 1986) with purified MOP cyclase.

\section{Growth studies.}

The inhibitory effect of MOP and its analogs on the growth of A. tumefaciens strains was tested semiquantitatively. Tested strains were grown for 2 days in LB containing the appropriate antibiotics. A $100-\mu \mathrm{l}$ volume of each culture was added to $2 \mathrm{ml}$ of premelted, warm $\left(45^{\circ} \mathrm{C}\right), 0.6 \%$ Nobel agar in water and vortexed briefly. The mixture was poured over the surface of plates containing solid ATNG or solid Luria agar (LA) me-

Table 4. Frequency of appearance of spontaneous mannopine (MOP)insensitive mutants from NT1(pRE10)

\begin{tabular}{lll}
\hline \multicolumn{2}{c}{ Growth condition } & \\
\cline { 1 - 2 } First culture $^{\mathbf{a}}$ & Second culture & \\
\hline Glucose & - & Frequency $^{\mathbf{c}}$ \\
Glucose + MOP & - & $9.62 \times 10^{-6}$ \\
Glucose & Glucose & $4.30 \times 10^{-3}$ \\
Glucose & Glucose + MOP & $8.24 \times 10^{-5}$ \\
Glucose + MOP & Glucose & $2.15 \times 10^{-2}$ \\
Glucose + MOP & Glucose + MOP & $7.73 \times 10^{-3}$ \\
\hline
\end{tabular}

${ }^{a}$ Cells were grown in liquid ATNG medium, with or without MOP, at a final concentration of $1 \mathrm{mM}$ at $28^{\circ} \mathrm{C}$ for $50 \mathrm{~h}$.

${ }^{\mathrm{b}}$ Cells from each of the cultures were washed twice and used to inoculate ATNG medium, with or without MOP. Cultures were grown at $28^{\circ} \mathrm{C}$ for $60 \mathrm{~h}$. After growth, cells were diluted serially and spread on solid ATNG media, with or without MOP, then further grown at $28^{\circ} \mathrm{C}$. After 4 days, the numbers of colonies appearing on the plates were counted.

${ }^{c}$ Frequency was calculated by dividing the titer of the MOP-insensitive colonies grown on solid ATNG medium containing MOP by the viable titer as assessed on solid ATNG medium. 
dium (Difco) supplemented with the appropriate antibiotics. After the top agar had solidified, a 1- $\mu$ l volume of solution composed of MOP, AGR, MOA, SOP, or MOP analogs at appropriate concentrations were spotted onto the surface of the medium. The plates were incubated at $28^{\circ} \mathrm{C}$ and observed visually for a 1-week period. The diameter of any zone of growth inhibition was measured, and the appearance of spontaneous mutants in the inhibition zone was noted.

\section{Growth inhibition studies.}

A 1-ml volume of NT1(pRE10) culture grown in ATNG medium for $30 \mathrm{~h}$ at $28^{\circ} \mathrm{C}$ was transferred to $30 \mathrm{ml}$ of ATNG medium containing $100 \mu \mathrm{g}$ of kanamycin per $\mathrm{ml}$ and $100 \mu \mathrm{g}$ of carbenicillin per ml. The culture was incubated at $28^{\circ} \mathrm{C}$ with shaking until the culture reached a density corresponding to a Klett value of approximately 20 with a red filter. The culture was split into two portions: MOP (with a final concentration of $1 \mathrm{mM}$ ) was added to one, and the two subcultures were incubated as above. Samples were taken at 2-h intervals, and population densities and viable cell numbers were measured by Klett colorimetry and dilution plating on ATNG plates, respectively.

\section{Estimating the presence}

of spontaneous MOP-insensitive mutants of NT1(pRE10).

A culture of NT1(pRE10) was grown to early exponential phase in ATNG medium. The culture was split into two 1-ml portions, MOP (at a final concentration of $1 \mathrm{mM}$ ) was added to one, and the two cultures were incubated as above to late exponential phase. Cells of the two sets of cultures were collected, washed twice with $0.9 \% \mathrm{NaCl}$, and resuspended in 1 $\mathrm{ml}$ of ATNG. A 10- $\mu$ l volume of cells from the two cultures was inoculated into a 1-ml volume of liquid ATNG medium and ATNG medium containing MOP at a final concentration of $1 \mathrm{mM}$. The four sets of cultures were grown to late expo- nential phase, and 10- $\mu$ l volumes of each culture were removed and spread on solid ATNG medium containing $1 \mathrm{mM}$ MOP. Volumes of appropriate dilutions also were spread on solid ATNG medium for viable cell counts. After incubation at $28^{\circ} \mathrm{C}$ for 4 days, the numbers of colonies were counted. The frequency of the appearance of MOP-insensitive mutants was obtained by dividing the titer derived from the number of colonies on solid ATNG medium containing MOP by the total viable titer derived from the number of colonies on solid ATNG medium without MOP.

\section{Uptake of MOP and its analogs.}

Uptake of MOP and its chemical analogs was estimated indirectly by measuring the disappearance of the substrates from liquid medium (Chilton and Chilton 1984). Tested strains were grown in $0.5 \mathrm{ml}$ of ATNG medium for $30 \mathrm{~h}$ at $28^{\circ} \mathrm{C}$ with shaking. Cells were collected by centrifugation and washed with a solution of $0.9 \% \mathrm{NaCl}$. The collected cells were resuspended in $100 \mu \mathrm{l}$ of ATN minimal medium containing MOP or one of its analogs at a final concentration of $1 \mathrm{mM}$ as the sole carbon source, and the cultures were incubated at $28^{\circ} \mathrm{C}$ with shaking. After $4 \mathrm{~h}$, cells were removed by centrifugation, and the supernatants were spotted on Whatman $3 \mathrm{MM}$ filter paper (Kent, U.K.). The filter paper strips were air dried, and the remaining substrate in the samples was visualized by the alkaline silver nitrate staining method, as described previously (Ellis et al. 1984). Opine utilizing and nonutilizing strains were included as positive and negative controls.

\section{DNA manipulations.}

Large- and small-scale isolations of plasmid DNA were performed by a rapid alkaline lysis procedure (Maniatis et al. 1982). Digestions with restriction endonucleases were performed as described by the manufacturers. Strains of A. tumefaciens were transformed by a freeze-thaw technique (Holsters

Table 5. Bacterial strains and plasmids used in this study

\begin{tabular}{|c|c|c|}
\hline Strains or plasmid & Relevant property or genotype $^{a}$ & Source or reference \\
\hline \multicolumn{3}{|l|}{ Strains } \\
\hline NT1 & pTiC58-cured derivative of C58, pAtC58 ${ }^{+}$. & E. W. Nester, University of Washington \\
\hline UIA5 & pTiC58- and pAtC58-cured derivative of C58. & Our collection \\
\hline UIA5(pRE10) & Constitutive mannopine (MOP) uptake. & Hong et al. 1993 \\
\hline NT1(pYDH208) & $\mathrm{MOP}^{+}$, agropine $(\mathrm{AGR})^{+}$, mannopinic acid $(\mathrm{MOA})^{\mathrm{i}}, \mathrm{Tc}^{\mathrm{r}}$ & Hong et al. 1993 \\
\hline $\mathrm{KYC} 8$ & Derivative of the octopine-type strain R10, ooxB::Tn5-gusA. & Cho et al. 1996 \\
\hline KYC11 & Derivative of the octopine-type strain $\mathrm{R} 10$, oocQ::Tn5-gusA. & Cho et al. 1996 \\
\hline KYC16 & Derivative of the octopine-type strain R10, ooxA ::Tn5-gusA. & Cho et al. 1996 \\
\hline \multicolumn{3}{|l|}{ Plasmids } \\
\hline pYDH208 & $\begin{array}{l}\text { Cosmid clone of pTi15955 conferring utilization of MOP, AGR, and } \\
\text { MOA on NT1, Tc }{ }^{\mathrm{r}} \text {. }\end{array}$ & Hong et al. 1993 \\
\hline pSaB4 & $\begin{array}{l}\text { Bam } \mathrm{HI} \text { fragment } 4 \text { of pTi15955 cloned in } \mathrm{pSa} 4 \Delta \mathrm{H} \text { and containing moc } \\
\text { genes for the degradation of MOP, AGR, and the AGR transport sys- } \\
\text { tem but lacking a MOP transport system, } \mathrm{Km}^{\mathrm{r}} \text {. }\end{array}$ & Kim and Farrand 1996 \\
\hline pSaB4::Tn5seq1 no. 135 & pSaB4 derivative with a Tn5seq1 insertion in $\operatorname{moc} C, \mathrm{Km}^{\mathrm{r}} \mathrm{Nm}^{\mathrm{r}}$. & Kim and Farrand 1996 \\
\hline pYDH208::Tn3HoHo1 no. 11 & pYDH208 derivative with a Tn3HoHo1 insertion in $m o c C, \mathrm{Cb}^{\mathrm{r}}$. & Hong et al. 1993 \\
\hline pYDH208::Tn5 no. 110 & $\begin{array}{l}\text { pYDH208 derivative with a Tn5 insertion in the MOP transport system, } \\
\mathrm{Km}^{\mathrm{r}} \text {. }\end{array}$ & Hong et al. 1993 \\
\hline $\mathrm{pSa}-\mathrm{C}$ & $\begin{array}{l}\text { Cointegrate of } \mathrm{pSa} 4 \Delta \mathrm{H} \text { and } \mathrm{pKS}-\mathrm{C} \text {, which is a derivative of pUC18 } \\
\text { containing } \operatorname{moc} C \text {. Constitutively expresses MocC (MOP oxidoreduc- } \\
\text { tase), } \mathrm{Cb}^{\mathrm{r}} \text {. }\end{array}$ & Kim et al. 1996 \\
\hline $\mathrm{pRE} 10$ & $\begin{array}{l}\text { Cointegrate of pRK } 415 \mathrm{~K} \text { and pUCE10, which is a derivative of pUC18 } \\
\text { containing EcoRI fragment } 10 \text { of pTi15955 coding for the entire mot } \\
\text { operon, } \mathrm{Km}^{\mathrm{r}} \text {. Constitutively expresses the MOP transport system. }\end{array}$ & Hong 1994 \\
\hline
\end{tabular}

${ }^{\mathrm{a}} \mathrm{Tc}^{\mathrm{r}}$, tetracycline resistant; $\mathrm{Km}^{\mathrm{r}}$, kanamycin resistant; $\mathrm{Nm}^{\mathrm{r}}$, neomycin resistant; $\mathrm{Cb}^{\mathrm{r}}$, carbenicillin resistant; $\mathrm{Cm}^{\mathrm{r}}$, chloramphenicol resistant. 
et al. 1978) or by electroporation (Cangelosi et al. 1991). Escherichia coli was transformed with the $\mathrm{CaCl}_{2}$ method (Maniatis et al. 1982).

\section{ACKNOWLEDGMENTS}

Portions of this work were supported by grants to S. K. Farrand from the Illinois Soybean Program Operating Board and the Biotechnology Research and Development Corporation and, in part, by a grant to K.-S. Kim from Sogang University. We thank W. S. Chilton of North Carolina State University for providing us with SOP, chrysopine, isochrysopine, and MOP analogs. We also thank W. Boos of the University of Konstanz, Germany, for invaluable discussions.

\section{LITERATURE CITED}

Baek, C. H., Lee, J. K., Farrand, S. K., and Kim, K.-S. 2000. The cryptic plasmid pAtC58 in nopaline-type Agrobacterium tumefaciens strain C58 encodes functions for the santhopine utilization. (Abstr.) The 100th General Meeting of the American Society for Microbiology, Los Angeles. I-117.

Barkulis, I. L. 1949. Growth inhibition of Eberthella typosa by certain carbohydrates and its release by mutation. J. Bacteriol. 58:103109.

Böck, A. and Neidhardt. 1966. Properties of a mutant of Escherichia coli with a temperature-sensitive fructose-1,6-diphosphate aldolase. J. Bacteriol. 92:470-476.

Cangelosi, G. A., Best, E. A., Martinetti, C., and Nester, E. W. 1991. Genetic analysis of Agrobacterium tumefaciens. Methods Enzymol. 145:177-181.

Chilton W. S., and Chilton, M.-D. 1984. Mannityl opine analogs allow isolation of catabolic pathway regulatory mutants. J. Bacteriol. 158:650-658.

Cho, K., Fuqua, C., Martin, B. S., and Winans, S. C. 1996. Identification of Agrobacterium tumefaciens genes that direct the complete catabolism of octopine. J. Bacteriol. 178:1872-1880.

Decker, K., Peist, R., Reidl, J., Kossmann, M., Brand, B., and Boos, W. 1993. Maltose and maltotriose can be formed endogenously in Escherichia coli from glucose and glucose-1-phosphate independently of enzymes of the maltose system. J. Bacteriol. 175:56555665 .

Dessaux, Y., Guyon, P., Farrand, S. K., Petit, A., and Tempé, J. 1986. Agrobacterium Ti and Ri plasmids specify enzymic lactonization of mannopine to agropine. J. Gen. Microbiol. 132:2549-2559.

Dessaux, Y., Tempé, J., and Farrand, S. K. 1987. Genetic analysis of mannityl opine catabolism in octopine-type Agrobacterium tumefaciens strain 15955. Mol. Gen. Genet. 208:301-308.

Dessaux, Y., Petit, A., Farrand, S. K., and Murphy, P. J. 1998. Opines and opine-like molecules involved in plant-Rhizobiaceae interactions. Pages 173-197 in: The Rhizobiaceae, Molecular Biology of Model Plant-Associated Bacteria. H. P Spaink, A. Kondorosi, and P. J. J. Hooykaas, eds. Kluwer Academic Publishers, Dordrecht, The Netherlands.

Ellis, J. G., and Murphy, P. J. 1981. Four new opines from crown gall tumors-Their detection and properties. Mol. Gen. Genet. 181:3643.

Ellis, J. G., Murphy, P. J., and Kerr, A. 1982. Isolation and properties of transfer regulatory mutants of the nopaline Ti plasmid pTiC58. Mol. Gen. Genet. 186:275-281.

Ellis, J. G., Ryder, M. M., and Tate, M. E. 1984. Agrobacterium tumefaciens $\mathrm{T}_{\mathrm{R}}$-DNA encodes a pathway for agropine biosynthesis. Mol. Gen. Genet. 195:466-473.

Fradkin, J. E., and Fraenkel, D. G. 1971. 2-Keto-3-deoxygluconate 6phosphate aldolase mutants of Escherichia coli. J. Bacteriol. 108:1277-1283.

Guyon, P., Petit, A., Tempé, J., and Dessaux, Y. 1993. Transformed plants producing opines specifically promote growth of opinedegrading agrobacteria. Mol. Plant-Microbe Interact. 6:92-98.

Hayman, G. T., and Farrand, S. K. 1988. Characterization and mapping of the agrocinopine-agrocin 84 locus on the nopaline Ti plas- mid pTiC58. J. Bacteriol. 170:1759-1767.

Hayman, G. T., Beck von Bodman, S., Kim, H., Jiang, P., and Farrand, S. K. 1993. Genetic analysis of the agrocinopine catabolic region of Agrobacterium tumefaciens $\mathrm{Ti}$ plasmid pTiC58, which encodes genes required for opine and agrocin 84 transport. J. Bacteriol. 175:5575-5584.

Holsters M., De Waele, D., Depicker, A., Messens, E., Van Montagu, M., and Schell, J. 1978. Transfection and transformation of Agrobacterium tumefaciens. Mol. Gen. Genet. 163:181-187.

Hong, S.-B. 1994. Physical, genetic and biochemical analysis of mannityl opine catabolism genes from the Agrobacterium Ti plasmid pTi15955. Ph.D. diss. University of Illinois, Urbana, IL, U.S.A.

Hong, S.-B., and Farrand, S. K. 1994. Functional role of the Ti plasmid-encoded catabolic mannopine cyclase in mannityl opine catabolism by Agrobacterium. J. Bacteriol. 176:3576-3583.

Hong, S.-B., Dessaux, Y., Chilton, W. S., and Farrand, S. K. 1993. Organization and regulation of the mannopine-cyclase associated opine catabolism genes in Agrobacterium tumefaciens strain 15955. J. Bacteriol. 175:401-410.

Irani, M. H., and Maitra, P. K. 1977. Properties of Escherichia coli mutants deficient in enzymes of glycolysis. J. Bacteriol. 132:398410.

Kim, K.-S. 1995. Molecular, genetic, and biochemical studies of mannityl opine utilization by Agrobacterium tumefaciens: Its evolutionary implications. Ph.D. diss. University of Illinois, Urbana, IL, U.S.A

Kim, K.-S., and Farrand, S. K. 1996. Ti plasmid-encoded genes responsible for catabolism of the crown gall opine mannopine by Agrobacterium are homologues of the T-region genes responsible for synthesis of this opine by the plant tumor. J. Bacteriol. 178:3285-3292.

Kim, K.-S., Chilton, W. S., and Farrand, S. K. 1996. A Ti plasmidencoded enzyme required for degradation of mannopine is functionally homologous to the T-region-encoded enzyme required for synthesis of this opine in crown gall tumors. J. Bacteriol. 178:32853292.

Maniatis, T., Fritsch, E. F., and Sambrook, J. 1982. Molecular Cloning: A Laboratory Manual. Cold Spring Harbor Laboratory, Cold Spring Harbor, NY, U.S.A.

Oger, P., Petit, A., and Dessaux, Y. 1997. Genetically engineered plants producing opines alter their biological environment. Nat. Biotechnol. 15:369-372.

Oger P., Kim, K.-S., Sackett, R. L., Piper, K. R., and Farrand, S. K. 1998. Octopine-type Ti plasmids code for a mannopine-inducible dominant-negative allele of traR, the quorum-sensing activator that regulates Ti plasmid conjugal transfer. Mol. Microbiol. 27:277-288.

Petit, A., Dessaux, Y., and Tempé, J. 1978. The Biological Significance of Opines I. A Study of Opine Catabolism by Agrobacterium tumefaciens. Pages 143-152 in: Procedures of the 4th International Conference on Plant Pathology Bacteria. M. Ride, ed. INRA, Angers, France.

Petit, A., David, C., Dahl, G. A., Ellis, J. G., and Guyon, P. 1983. Further extension of the opine concept: Plasmids in Agrobacterium rhizogenes cooperate for opine degradation. Mol. Gen. Genet. 90:204-214.

Savka, M. A., and Farrand, S. K. 1997. Modification of rhizobacterial populations by engineering bacterial utilization of a novel plantproduced resource. Nat. Biotechnol. 15:363-368.

Tempé, J., Guyon, P., Tepfer, D., and Petit, A. 1979. The role of opines in the ecology of the Ti plasmids of Agrobacterium. Pages 353-363 in: Plasmids of Medical, Environmental, and Commercial Importance. K. N. Timmis and A. Pühler, eds. Elsvier/North Holland Biomedical Press, Amsterdam.

Vaudequin-Dransart, V., Petit, A., Poncet, C., Ponsonnet, C., Nesme, X., Jones, J. B., Bouzar, H., Chilton, W. S., and Dessaux, Y. 1995. Novel Ti plasmids in Agrobacterium strains isolated from fig tree and chrysanthemum tumors and their opinelike molecules. Mol. Plant-Microbe Interact. 8:311-321.

Vaudequin-Dransart, V., Petit, A., Chilton, W. S., and Dessaux, Y. 1998. The cryptic plasmid of Agrobacterium tumefaciens cointegrates with the Ti plasmid and cooperates for opine degradation. Mol. Plant-Microbe Interact. 11:583-591.

Veluthambi, K., Krishnan, M., Gould, J. H., Smith, R. H., and Gelvin, S B. 1989. Opines stimulate induction of the vir genes of the Agrobac- 
terium tumefaciens Ti plasmid. J. Bacteriol. 171:3696-3703.

Wilson, M., Savka, M. A., Farrand, S. K., and Lindow, S. E. 1995.

Altered epiphytic colonization of mannityl opine-producing transgenic tobacco plants by a mannityl opine-catabolizing strain of
Pseudomonas syringae. Appl. Environ. Microbiol. 61:2151-2158.

Zhu, J., Oger, P. M., Schrammeuer, B., Hooykaas, P. J. J., Farrand, S

K., Winans, S. C. 2000. The bases of crown gall tumorigenesis. J. Bacteriol. 182:3885-3895. 